\title{
Effect of trachea stiffness on tumor distribution in papillary thyroid microcarcinoma
}

\author{
HUA ZHANG ${ }^{1 *}$, TAIYANG LI ${ }^{2 *}$, XILONG DU ${ }^{3}$, QIHANG LI ${ }^{2}$, BO HUO ${ }^{2}$, RUI JIN ${ }^{1}$ and PING LI ${ }^{1}$ \\ ${ }^{1}$ Department of Maxillofacial and Ear Nose and Throat Oncology, Tianjin Medical University Cancer Institute and \\ Hospital, National Clinical Research Center for Cancer, Key Laboratory of Cancer Prevention and Therapy, \\ Tianjin 300020; ${ }^{2}$ Beijing Institute of Technology, School of Aerospace Engineering, Beijing 100081; \\ ${ }^{3}$ Beijing Joy Gene Tech Co., Ltd., Beijing 100021, P.R. China
}

Received September 16, 2020; Accepted April 9, 2021

DOI: $10.3892 / \mathrm{ol} .2021 .12779$

\begin{abstract}
Biomechanical factors play an important role in tumor distribution, epithelial-mesenchymal transition (EMT), invasion and other important processes. Despite fewer reports investigating biomechanical function in papillary thyroid carcinoma (PTC), a large number of PTC cases are located close to the trachea and the majority of advanced cases of PTC have been associated with invasion of the trachea. However, the effect of trachea stiffness on PTC distribution and growth remains unknown. To clarify this issue, two types of PTC cells (TPC-1 and KTC-1) were seeded on a substrate with different stiffness to observe cell proliferation and movement. To identify the effect of trachea stiffness on the thyroid, two thyroid lobes (left and right) were evenly divided into interior (close to the trachea) and lateral (away from the trachea) parts, based on the vertical line between the trachea and thyroid lateral margin with different von Mises stress values. As PTC originates from papillary thyroid microcarcinoma (PTMC) with a maximum diameter of $<1 \mathrm{~cm}$, the present study selected PTMC as the study subject to reflect initial PTC distribution in the thyroid. The association between the percentage of PTMC distribution in different parts of the thyroid and von Mises stress values was analyzed. Both PTC cells exhibited stronger proliferation and mobility on the stiff substrate compared with that on the soft substrate. Furthermore, the results of finite element analysis revealed that the von Mises stress values of the interior
\end{abstract}

Correspondence to: Dr Rui Jin or Dr Ping Li, Department of Maxillofacial and Ear Nose and Throat Oncology, Tianjin Medical University Cancer Institute and Hospital, National Clinical Research Center for Cancer, Key Laboratory of Cancer Prevention and Therapy, Tianjin 300020, P.R. China

E-mail:245085019@qq.com

E-mail: pingli@tmu.edu.cn

${ }^{*}$ Contributed equally

Key words: papillary thyroid microcarcinoma, trachea stiffness, distribution, biomechanical genes parts of the trachea were notably higher compared with that in the lateral parts. PTMC distribution in the interior trachea was notably greater compared with that in the lateral section. There was also an observed association between von Mises stress values and PTMC distribution. In addition, the results of RNA-sequencing and reverse transcription-quantitative PCR demonstrated that three biomechanical genes were overexpressed in PTMC located in the interior section compared with that in adjacent normal tissue, and the related signaling pathways were also activated in these tissues. On the whole, these results indicated that trachea stiffness may supply a suitable biomechanical environment for PTMC growth, and the related biomechanical genes may serve as novel targets for PTMC diagnosis and prognostic estimation.

\section{Introduction}

Matrix stiffness, resulting from abundant matrix protein deposition and crosslinking, as one of the important biomechanical factors, plays important roles in tumor progression, including epithelial-mesenchymal transition (EMT), motility, distribution, invasion, metastasis and stemness in tumors $(1,2)$. For example, melanoma cells could migrate towards higher extracellular matrix (ECM) densities or stiffer areas of the substratum. The increased matrix stiffness, following thermal ablation, could promote the progression of residual hepatocellular carcinoma by a stiffness-dependent regulation of ERK phosphorylation (3). The inhibition of the ECM stiffness in the mouse mammary gland caused tumor cells to revert to a normal epithelial phenotype, which could be characterized by reduced invasion and proliferation (4).

Thyroid cancer is responsible for 586,000 cases worldwide, ranking in 9th place for incidence in 2020 (5). Papillary thyroid carcinoma (PTC) is one of the most common endocrine malignancies in worldwide, research has demonstrated a continuous increase in the global incidence of thyroid cancer over the past two decades (6). Despite a number of studies on the biochemical alterations in PTC $(7,8)$, the developmental processes of PTC remain to be fully determined. To the best of our knowledge, reports on the biomechanical influence and genetic alterations in PTC are limited. Very recently, Jasim et al (9) found that thyroid nodule location was an 
independent risk factor in predicting the risk of thyroid cancer. Normally, isthmic nodules carry the highest risk of cancer diagnosis and lower lobe nodules carry the lowest risk. Naturally, the isthmus is closely attached to the trachea with a relative stiffness. Furthermore, a number of studies have demonstrated that the trachea is the most common site of invasion, with an incidence rate of $35-60 \%$ in patients with PTC and tumor invasion, followed by the larynx and esophagus $(10,11)$; the incidence rate per site was as follows: $77 \%$ for recurrent laryngeal nerve, $55 \%$ for the trachea, $4 \%$ for the larynx and $15 \%$ for the esophagus (12), while $<4 \%$ of patients exhibited vein and soft tissue invasion. All these results indicated that PTCs occur more frequently around the trachea where the tissue stiffness is higher; however, the association between tissue stiffness and tumor distribution remains unclear. It is thus, crucial to investigate the effect of trachea stiffness on PTC distribution and to determine its association with tumor prevention, diagnosis and treatment.

The present study constructed trachea stiffness analysis technology and quantitatively evaluated the genetic performance of PTC, with adjacent normal tissue as a control to identify the association between trachea stiffness and PTC distribution to provide novel diagnostic and treatment markers for PTC. Combining in vitro and in vivo analyses, elucidating the association between trachea stiffness and PTC distribution may provide new insight into PTC development and lead to the design of new treatment strategies.

\section{Materials and methods}

Identification of PTMC tissue stiffness using nanoindentation technology. This was detected using a nanoindentation instrument (Hysitron TI980 TriboIndenter, Bruker Corporation) and its association between the displacement and the experimental load was analyzed.

Cell experiments on a substrate with different stiffness levels. The TPC-1 and KTC-1 cell lines, both derived from humans, were purchased from the Cell Bank of the Chinese Academy of Sciences. Both the cell lines were maintained in RPMI-1640 medium (Invitrogen; Thermo Fisher Scientific, Inc.) supplemented with $10 \%$ fetal bovine serum (Invitrogen; Thermo Fisher Scientific, Inc.), $100 \mathrm{IU} / \mathrm{ml}$ penicillin (Gibco; Thermo Fisher Scientific, Inc.) and $100 \mu \mathrm{g} / \mathrm{ml}$ streptomycin (Gibco; Thermo Fisher Scientific, Inc.), at $37^{\circ} \mathrm{C}$ in a humidified incubator with $5 \% \mathrm{CO}_{2}$.

The cells were then seeded in a 6-well plate, at a concentration of $1 \times 10^{5}$ cells/well with 10,40 and $60 \mathrm{kPa}$ substrate for $12 \mathrm{~h}$, in which the substrate was prepared according to a previous report (13). Subsequently, the cells were scratched vertically with a $100-\mu l$ pipette tip the following day. At $48 \mathrm{~h}$, the cells were counted under an inverted phase-contrast microscope in 5 random fields, after washing twice with PBS and placed in serum-free culture medium.

The cells of $1 \times 10^{5} / \mathrm{ml}$ were seeded in six-well culture plates with different substrate stiffness. The $10 \mathrm{kPa}$ stiffness group was served as the control. After 24-h cell culture, cells were trypsinized for cell counting using Coulter counter every $12 \mathrm{~h}$, then re-suspended and reseeded in dishes till $72 \mathrm{~h}$. The above experiments were repeated three times for each group.
Finite element analysis (FEA) for the thyroid and its surrounding tissue

Construction of the $3 D$ volume model. Computed tomography (CT) data of the neck was collected from 7 patients with PTC for FEA (Department of Maxillofacial and Ear Nose and Throat Oncology, Tianjin Medical University Cancer Institute and Hospital). The 3D image data of the patients with PTC were captured using CT technology (ScanXmate-E090; Comscantecno Co., Ltd.). Following air calibration, X-ray exposure in the neck of each patient was performed with a view field of $25 \times 25 \mathrm{~cm}$ and an axial scan, at a thickness of $2.5 \times 2.5 \mathrm{~mm}$ interval. The CT data was used to construct the $3 \mathrm{D}$ polygonal stereolithography (STL) model of the neck in each case. A part of the STL model, with $\sim 2.5 \mathrm{~mm}$ anterior-posterior thickness, was retrieved from the whole model to obtain a segment representing the region of interest, including the loading site. This segmented STL model was converted into computer-aided design software (Catia V5R18; Dassault Systems) to analyze the model in detail and examine minute irregularities. Finally, the 3D volume model was constructed using Ansys finite element software (Ansys version 11.0; ANSYS, Inc.), which was meshed by 10 -nodes quadratic tetrahedral element with 3 degrees of freedom.

Definition of material parameters. The thyroid gland was set as viscoelastic and isotropic material, with an initial Young's modulus of $34.85 \mathrm{MPa}$ and Poisson's ratio of 0.49 (14). The trachea was defined as isotropic elastic material with a Young's modulus of 3.33 MPa and Poisson's ratio of 0.49 (15). The density of the thyroid was set as $1,150 \mathrm{~kg} / \mathrm{m}^{3}$, and the trachea was considered as cartilage only with a density of $1,400 \mathrm{~kg} / \mathrm{m}^{3}$, which was obtained using the density measurement function of Mimics version 8.1 software (Materialise; https:/www. materialise.com/).

Papillary thyroid microcarcinoma (PTMC) distribution in the thyroid and its clinical information. The present clinical study included 998 patients who were diagnosed with PTMC by a pathologist following surgery at the Tianjin Medical University Cancer Hospital (TJMUCH) between 1st June 2016 and 1st December 2016; 709 patients had a single tumor and 289 patients had multifocal disease. The mean age was $48.2 \pm 11.3$ years and there were 261 males and 737 females. The present study was conducted according to the principles outlined in the Helsinki Declaration and was approved by the Research Ethics Committee at TJMUCH (no. 2018090). Written informed consent was provided by all the patients. The clinicopathological data, including sex, age and the presence of thyroiditis, were also collected. All the patients were subjected to total thyroidectomy or unilateral thyroidectomy according to the National Comprehensive Cancer Network guidelines (version 2016) (16,17); cases in which the tumor was close to the tracheal region were marked as 'interior', and those with tumors 2-4 cm away from the trachea were marked as 'lateral'.

RNA extraction, sequencing and preprocessing. A total of 33 samples, each containing PTMC located in interior regions and adjacent normal tissue, were collected for RNA-seq analysis (3 samples) and RT-qPCR (30 samples). A total of 10 samples, 
including multifocal PTMC located in the interior and lateral regions, were collected for RT-qPCR for comparing differences in gene expression between PTMC located in different regions of the trachea. All tissue samples were obtained by thyroidectomy and stored at $-80^{\circ} \mathrm{C}$ until further use. RNA sequencing (RNA-seq) and subsequent analysis was performed by the BGI-Shenzhen Company (http://www.genomics.cn/en/).

Identification of differentially expressed genes (DEGS) using RNA-seq analysis. Gene expression levels of the transcripts were measured using the reads per kilobase of transcript, per million mapped reads method. Subsequently, the edgeR package (edgeR 3.14.0) (18) tool was utilized to identify the DEGs between the PTMC and adjacent normal tissue from 3 samples of PTMC. During the differential analysis, the negative binomial model was used to calculate the significance of the differentially expressed mRNAs, followed by the adjustment of P-values using the Benjamini Hochberg method (19). The cut-off values of the DEG selection was a false discovery rate adjusted $\mathrm{P}<0.05$ and $\log _{2}$ fold change $I \geq 1$. These results were determined based on the comparison with The Cancer Genome Atlas (TCGA). Venn diagram analysis was performed using online software at the following URL: http://bioinformatics.psb.ugent. be/webtools/Venn/.

Functional enrichment analysis and construction of the protein-protein interaction (PPI) network using TCGA. To further investigate the functions and pathways of the DEGs, Gene Ontology (GO; http://www.geneontology.org/) and Kyoto Encyclopedia of Genes and Genomes (KEGG) (www. kegg.jp) pathway enrichment analyses were performed using 'Term Finder' (https://yeastgenome.org/goTermFinder), with a threshold corrected $\mathrm{P} \leq 0.05$ for the identification of significant GO terms and pathways using default settings. Furthermore, Search Tool for the Retrieval of Interacting Genes/Proteins (http://string-db.org/) was used to further investigate the associations in the DEGs, at the protein level (20). The criterion for the construction of the PPI network was based on the confidence score $\geq 0.90$. The Cfinder software (version 2.0.6, http://www.cfinder.org/) was used to extract the functional modules of the PPI network with default parameters (21).

RT-qPCR. 30 samples tissues, including PTMC and adjacent normal tissue, were selected for RT-PCR test. Three biomechanical genes, which were overexpressed from RNA-seq analysis were analyzed using RT-qPCR for validation and the TransStart Top Green qPCR SuperMix (Beijing Transgen Biotech Co., Ltd.). The PCR was performed using the ABI7500 Real-Time qPCR System (Applied Biosystems; Thermo Fisher Scientific, Inc.) and the following thermocycling conditions: Initial denaturation at $95^{\circ} \mathrm{C}$ for $20 \mathrm{sec}$, followed by 40 cycles of $95^{\circ} \mathrm{C}$ for $15 \mathrm{sec}$, annealing at $60^{\circ} \mathrm{C}$ for $30 \mathrm{sec}$ and extension at $70^{\circ} \mathrm{C}$ for $90 \mathrm{sec}$. The specificity of RT-qPCR was examined using the dissociation curve and the relative expression of the selected DEGs was normalized with the $18 \mathrm{~S}$ rRNA gene. The cycle threshold $(\mathrm{Cq}) 2^{-\Delta \Delta \mathrm{Cq}}$ method was used to calculate the relative expression level (22). All the gene specific primers used are listed in Table SI.
Statistical analysis. All the data are presented as the mean \pm SEM. Gene expression analysis was based on sex, age or thyroiditis. For the data from the patients with PTC, the categorical variables were analyzed using a $\chi^{2}$ or Fisher's exact test. Continuous variables were analyzed using a paired and unpaired Student's t-tests as appropriate. Comparisons of $>2$ groups were performed using ANOVA followed by Bonferroni's post hoc test. $\mathrm{P}<0.05$ was considered to indicate a statistically significant difference. ImageJ software (version 1.42; National Institutes of Health) was used for wound healing analysis. Origin software (version 9.0; OriginLab Corporation) was used for all other data analysis.

\section{Results}

PTMC stiffness. Thyroid images were captured and used to construct thyroid model (Fig. S1). When the load reaches the maximum value (hmax), the displacement also reaches the maximum value (Pmax), that is the maximum indentation depth. After unloading, the displacement finally returns to a fixed value (S). At this time, the depth is called the residual indentation depth (hr), that is the permanent plastic deformation left by the indenter on the sample (Fig. S2A). As the load reaches the maximum value of $0.743 \mathrm{mN}$, the displacement reached the maximum indentation depth of $984.63 \mathrm{~nm}$; after unloading, the displacement finally returned to the residual indentation depth of $881.36 \mathrm{~nm}$ (Fig. S2C). The results showed that PTMC tissue stiffness ranged from 20-70 kPa due to calcification or fibrosis (Fig. S2D).

Characterizations of the cell migratory and proliferative abilities on different substrates. To evaluate the migratory and proliferative abilities of the PTC cells on different substrates, these characteristics were analyzed using different experiments. As shown in Fig. 1, the scratch areas in the control and $60 \mathrm{kPa}$ groups were significantly narrower compared with that in the 10 and $40 \mathrm{kPa}$ groups for the TPC-1 (Fig. 1A) and KTC-1 (Fig. 1B) cell lines, suggesting a stronger migratory ability of these cells on the stiffer substrate compared with that on the softer substrate $(\mathrm{P}<0.001$; Fig. $1 \mathrm{C}$ and $\mathrm{D})$. Furthermore, the density of the TPC-1 cells on the $60 \mathrm{kPa}$ substrate following $72 \mathrm{~h}$ of culture was $7.19 \pm 0.2 \times 10^{5} / \mathrm{ml}$. However, the cell densities were $2.88 \pm 0.06 \times 10^{5} / \mathrm{ml}$ and $1.2 \pm 0.03 \times 10^{5} / \mathrm{ml}$ on the 40 and $10 \mathrm{kPa}$ substrates, respectively (Fig. 1E). It was notably different among the different stiffness groups compared with $10 \mathrm{kPa}$ groups, $(\mathrm{P}<0.001$; Fig. 1F). For the KTC-1 cell line, similar results were obtained, with densities of $7.36 \pm 0.29 \times 10^{5} / \mathrm{ml}$, $4.59 \pm 0.11 \times 10^{5} / \mathrm{ml}$ and $1.75 \pm 0.05 \times 10^{5} / \mathrm{ml}$ on the 60,40 and $10 \mathrm{kPa}$ substrates, respectively (Fig. 1G). A statistically significant difference was observed among these groups $(\mathrm{P}<0.001$; Fig. $1 \mathrm{H}$ ), which indicated a stronger proliferation ability of the cells on the stiffer substrate compared with that on the softer substrate.

Uneven stress distribution between the thyroid section close to and away from the trachea. Von Mises stress, an equivalent stress, was selected to reveal the distribution of elastic stress in the biological tissue. As shown in Fig. 2C, the von Mises stress in the thyroid was mainly produced by stiffness and the deformation of the trachea, which was significantly higher 

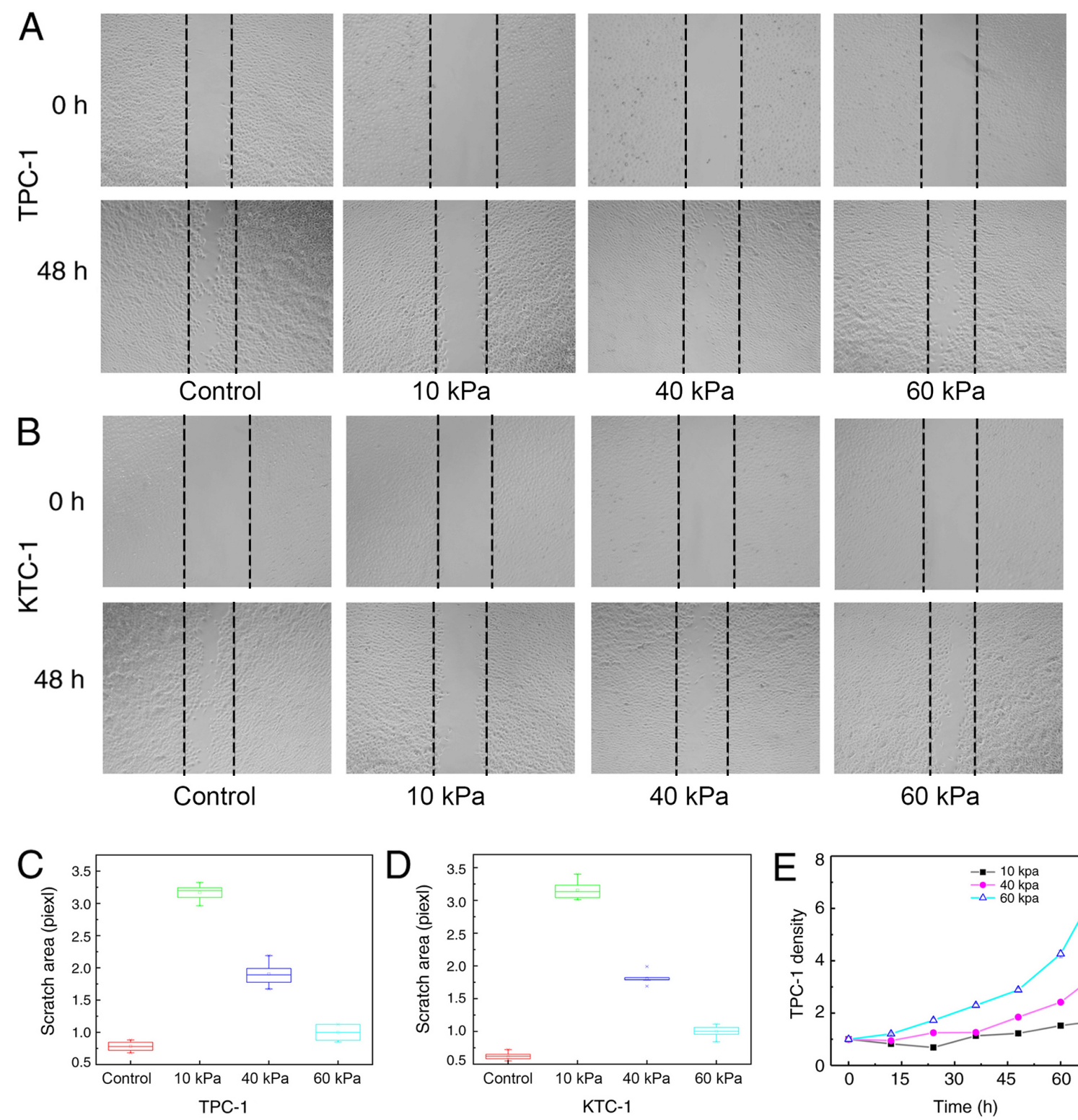

$10 \mathrm{kPa}$

$40 \mathrm{kPa}$

$60 \mathrm{kPa}$

Control
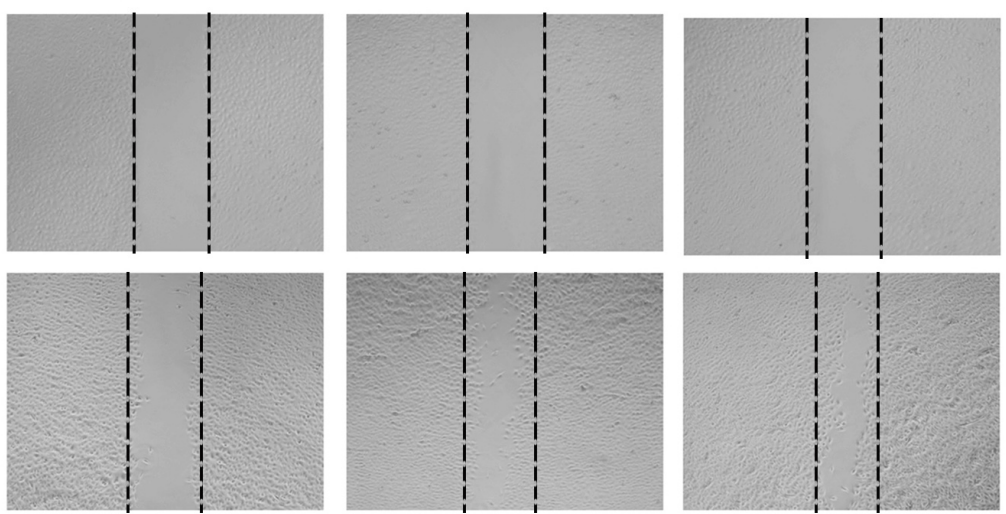

$40 \mathrm{kPa}$

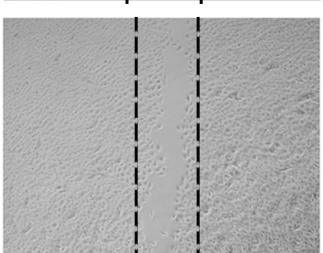

$10 \mathrm{kPa}$
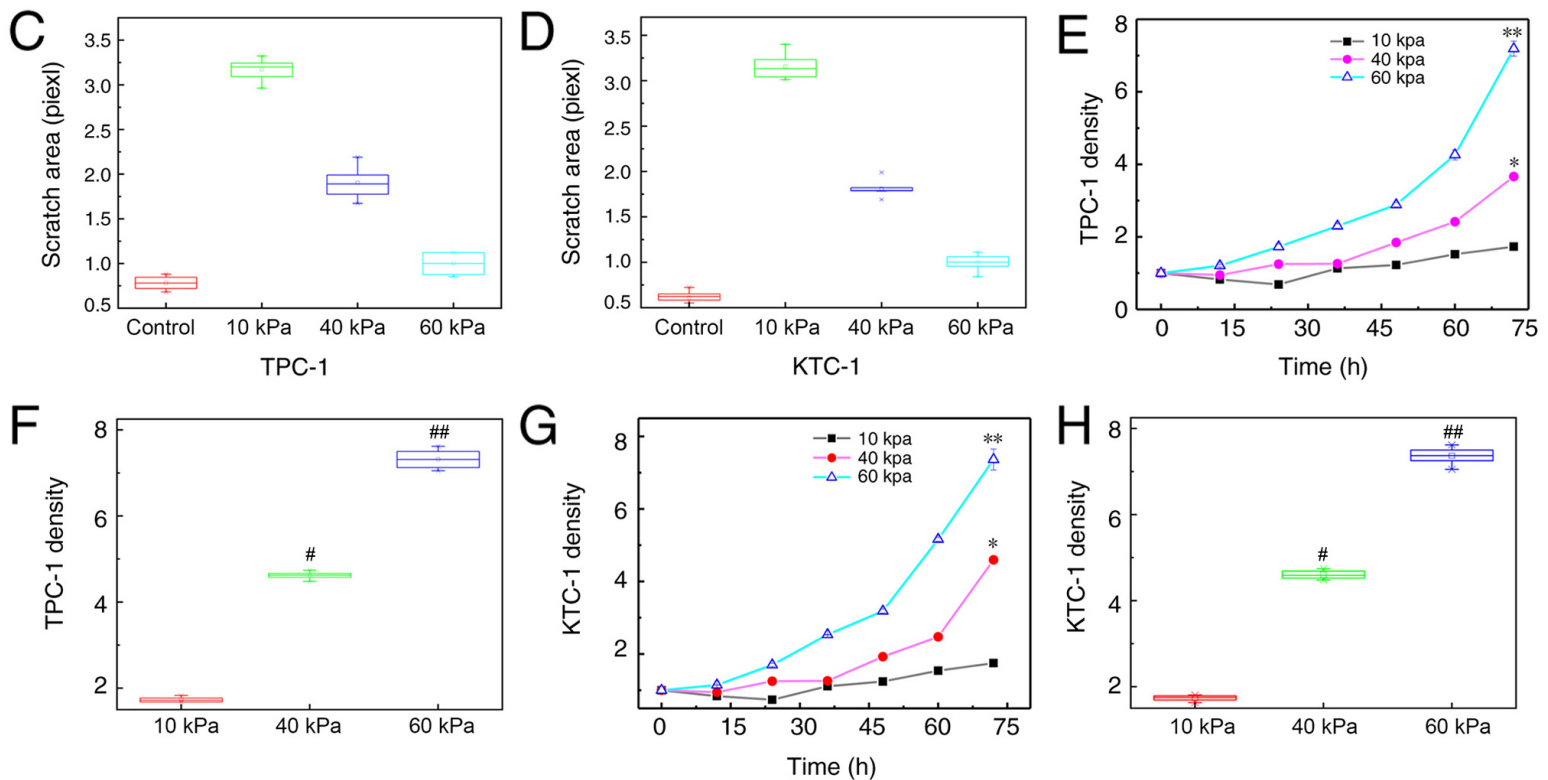

Figure 1. Effect of substrate stiffness on the migration and growth of the TPC-1 and KTC-1 cells. Wound healing was performed following culture of the (A) TPC-1 and (B) KTC-1 cells on the substrate with different stiffness for $48 \mathrm{~h}$. The scratch area (Piexl) results from the control, 10,40 and $60 \mathrm{kPa}$ groups of the (C) TPC-1 and (D) KTC-1 cells. (E) Growth curves of the TPC-1 cells on substrates with different stiffness. (F) TPC-1 density (1x10 $\mathrm{mmol} / \mathrm{l})$ following culture for $72 \mathrm{~h}$ in the 10, 40 and $60 \mathrm{kPa}$ groups. (G) Growth curves of the KTC-1 cells on substrates with different stiffness. (H) KTC-1 density following culture for $72 \mathrm{~h}$ in the 10,40 and $60 \mathrm{kPa}$ groups. ${ }^{\#} \mathrm{P}<0.05$ compared with $10 \mathrm{kPa}$ group. ${ }^{\# \#} \mathrm{P}<0.05$ represent $60 \mathrm{kPa}$ compared with 10 and $40 \mathrm{kPa}$ groups. ${ }^{*} \mathrm{P}<0.05$ compared with $10 \mathrm{kPa}$ group in 72 hours for cells density. ${ }^{* *} \mathrm{P}<0.0560 \mathrm{kPa}$ compared with 10 and $40 \mathrm{kPa}$ groups in 72 hours for cells density.

in the 'interior' compared with that in the 'lateral' regions $(10.18 \pm 3.35$ vs. $7.71 \pm 2.89 \mathrm{kPa}$, respectively; $\mathrm{P}<0.05)$.

Characterizations of PTMC distribution on different regions. As shown in Table I, the percentages of PTMC distribution in the 'interior' and 'lateral' sections were 68.58 and $31.42 \%$ in males, and 66.22 and $33.78 \%$ in females, respectively. There were no statistically significant differences in the parameters of sex, age or thyroiditis $(\mathrm{P}>0.05)$. The number of PTMC cases in the 'interior' and 'lateral' sections was 916 and 458, 
Table I. PTMC distribution in thyroid.

\begin{tabular}{lcc}
\hline Characteristic & Interior, $n(\%)$ & Lateral, n $(\%)$ \\
\hline Sex & & \\
$\quad$ Male & $179(68.58)$ & $82(31.42)$ \\
Female & $737(66.22)$ & $376(33.78)$ \\
Age, years & & \\
$\geq 50$ & $403(66.50)$ & $203(33.50)$ \\
$\leq 50$ & $513(66.79)$ & $255(33.21)$ \\
Thyroiditis & & $104(31.42)$ \\
Yes & $227(68.58)$ & $354(33.94)$ \\
No & $689(66.06)$ & $P>0.05$ \\
\hline
\end{tabular}

Data are divided into three groups depending on sex, age and thyroiditis, and each group including interior and lateral, respectively.

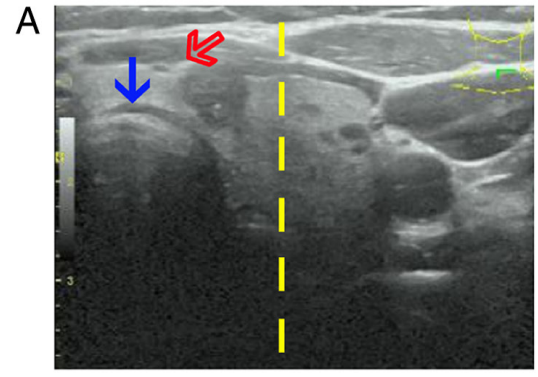

PTMC in interior

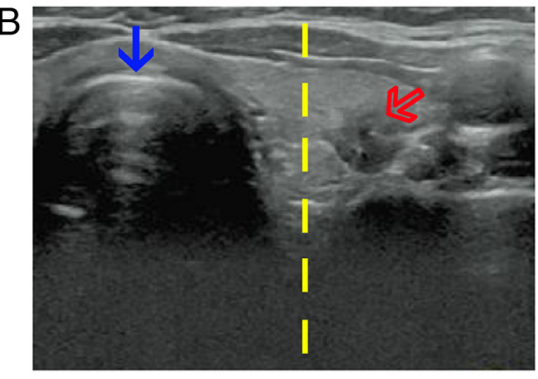

PTMC in lateral

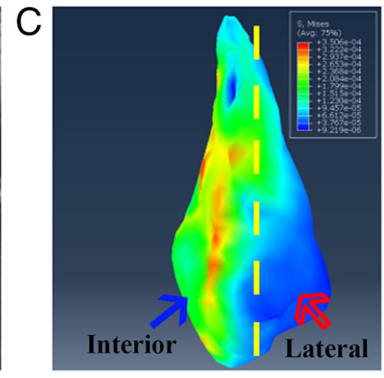

Figure 2. PTMC distribution feature and its association with von Mises stress. The unilateral thyroid gland lobe was equally divided by the yellow dash line. PTMC located closed to trachea and far from trachea were defined as (A) 'interior' or (B) 'lateral', respectively. The blue arrow indicates the trachea and the red arrow indicates PTMC. (C) The contour graph of von Mises stress in the thyroid gland with finite element analysis. The red color represents higher stress level and blue color represents lower stress level. PTMC, papillary thyroid microcarcinoma.

respectively, suggesting a possible association between PTMC distribution and Von Mises stress in the thyroid.

Biomechanical gene expression in PTMC and adjacent normal tissues. To evaluate biomechanical gene expression in different tissues, including PTMC and adjacent normal tissue, a total of 18,154 genes were detected from gene expression profiling. Flow diagram analysis of all the expressed genes indicated that 15,743 $(86.7 \%$ ) were expressed in all 3 samples for RNA-seq (Fig. 3B). The heatmap revealed the relative gene expression level in different samples (Fig. 3B). Based on the criteria, 1,504 genes were identified as DEGs between the PTC and adjacent normal tissues, of which 794 were upregulated and 710 were downregulated (Fig. S4A). In addition, the results of RT-qPCR indicated that the overexpressed genes in PTC were biomechanical sensors, including Piezo2, TRPV4 and $C D H 3$ (Fig. 4A), ECM stiffness and EMT related genes, including CLDN1, CLDN16, Runx2, Twist and G3BP3 (Fig. 4B) compared with that in the lateral normal tissues. However, some of the G-protein-related genes, including $A D O R A 1$ and $G A B B R 2$, exhibited a higher expression in PTC compared with that in adjacent normal tissues; however, there was no notable difference in the expression of RHOA among the groups (Fig. 4C). The expression level of these genes did not exhibit any significant difference by sex, age or thyroiditis, as shown in Fig. 5.
With respect to the Piezo2, CHD3, Runx2 and Twistl genes, the expression levels in PTMC, located in the interior section were notably higher compared with that in the lateral regions (Fig. S3).

Characterizations of the biomechanical functions and pathways in PTC. GO functional enrichment analysis in PTC, in comparison with TCGA, revealed the top $10 \mathrm{GO}$ enrichment terms for tumors for 'biological processes' (BP) (Fig. 4D), 'cellular component' (CC) (Fig. 4E) and 'molecular function' (MF) (Fig. 4F).

The upregulated genes were significantly enriched in 'cell adhesion' (GO: 0007155), 'immune system process' (GO: 0002376), ‘ECM organization' (GO: 0030198), ‘osteoclast formation', 'anatomical structure development' (GO: 0048856), ‘single-organism cellular process' (GO: 0044763), 'response to stress' (GO: 0006950), 'cell proliferation' (GO: 0008283) (Fig. S4).

The most notable BP terms of the downregulated genes were 'anatomical structure development' (GO: 0048856), 'single-organism developmental process' (GO: 0044767), 'cell differentiation' (GO: 0030154), 'cellular developmental process' (GO: 0048869), 'extracellular matrix organization’ (GO: 0030198), ‘cell morphogenesis' (GO: 0000902), 'cell adhesion' (GO: 0007155) (Fig. S4C). KEGG pathway 

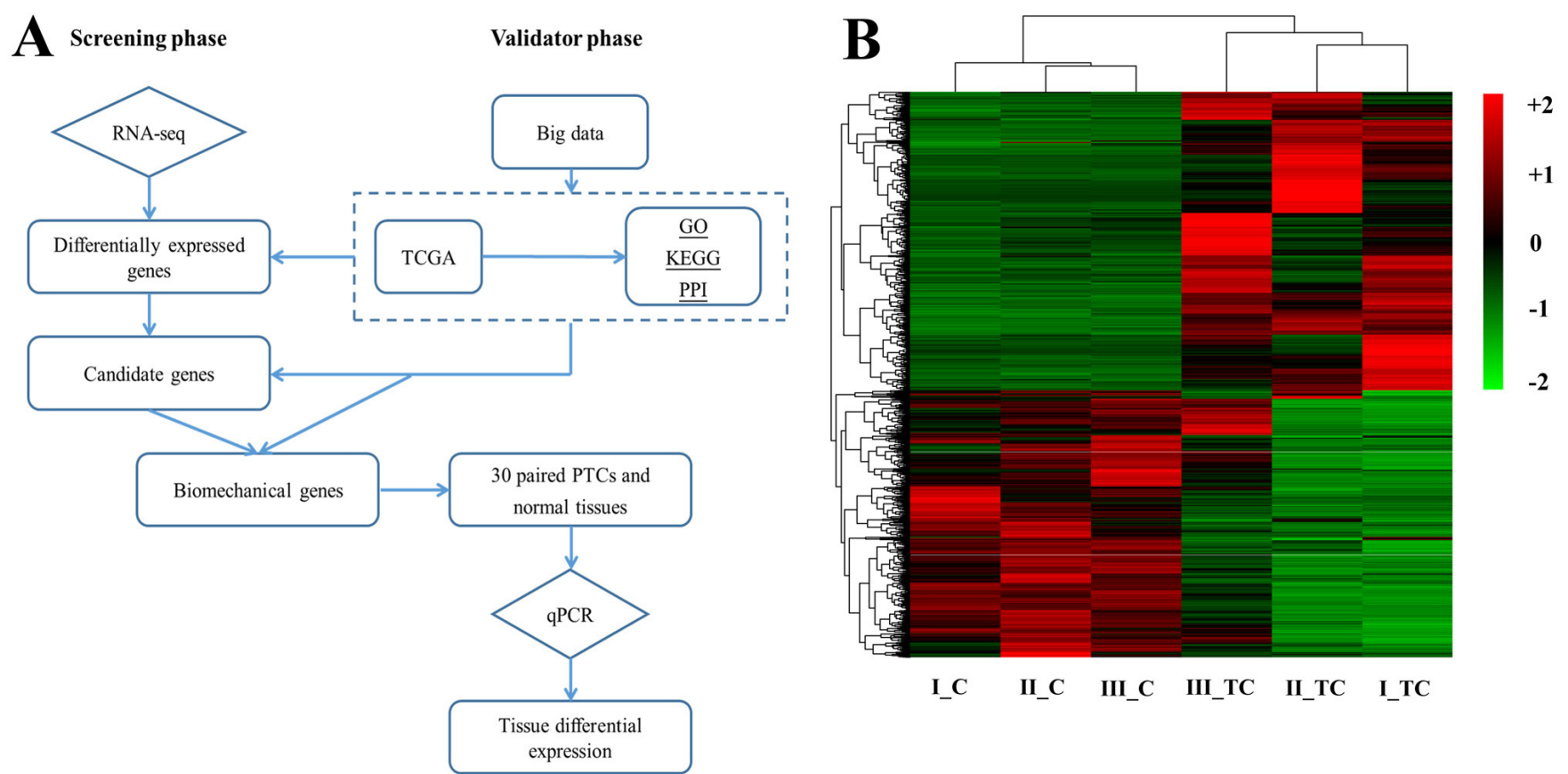

Figure 3. Differential gene expression between PTMC and adjacent normal tissue using RNA-sequencing and reverse transcription-quantitative PCR. (A) Flow diagram of the study design illustrating how the patients and controls were divided into screening and validation phase of the study. (B) Hierarchical clustering and heat map of the differentially expressed genes in the PTC and adjacent normal tissue based on the expression levels. The red and green regions represent the relatively up- and downregulated gene expression levels, respectively. The X-axis represents the control and PTC samples, while the Y-axis represents different genes. PTC, papillary thyroid carcinoma; TCGA, The Cancer Genome Atlas.

annotation revealed that signal transduction and immune system-related genes mainly participated in PTMC (Fig. S4D).

\section{Discussion}

Similar to other types of endothelial cancer, PTC cells exhibit a stronger migratory ability and favor growth on a stiffness substrate in vitro (23-26). As shown in the direct performance of this feature in the thyroid, $>60 \%$ of PTMC cases are located close to the trachea, which is a relatively stiff organ. Jasim et al (9) reported that thyroid tumors located on the isthmus exhibited the highest possibility of malignancy, followed by upper and middle thyroid nodules. In the present study, from the FEA of the thyroid, the von Mises stress values of the isthmus and upper section close to the trachea were higher compared with that away from the trachea (Fig. 2C). Therefore, there may be an association between PTC distribution and stiffness, indicating a related differential gene expression in PTC.

RNA-seq and RT-qPCR determined that three biomechanical genes exhibited up- or downregulated expression in PTC compared with that in adjacent normal tissue, and the related signaling pathways were enriched. For example, Piezo2 is commonly recognized as an important mechanotransduction channel participating in proprioception $(27,28)$, pain (29) and lung airway stretching (30). It also serves as a novel regulator of glioma angiogenesis and hyperpermeability; knocking down the expression of Piezo2 using small interfering RNA significantly inhibited the growth of glioma in both in vivo and in vitro experiments (31). The overexpression of Piezo2 was detected in PTC, but not in adjacent normal tissue (Fig. 4A), which warrants further investigation.
E-cadherin, as an important marker regulated by cadherin $3(\mathrm{CDH} 3)$ in epithelial cells, is overexpressed during thyroid development (32). $C D H 3$ is involved in various cellular activities, including cell adhesion, motility, invasion and the signaling of tumor cells and organ development (33). In breast cancer, $C D H 3$ has been found to be overexpressed in high-grade tumors and is a well-established indicator of aggressive tumor behavior (34). The results of the present study demonstrated that $\mathrm{CDH} 3$ was overexpressed in PTC (Fig. 4A). Furthermore, its expression in PTMC located close to the trachea was notably higher compared with that in regions further away from the trachea; its function warrants further exploration. In addition, other trachea stiffness-related genes, such as Piezo2, and biomechanical and EMT-related genes, including Runx2 and Twist 1 , also showed higher expression in PTMC located interiorly than laterally. Due to the important function of these genes in tumor metabolism, their effects on PTMC located in different locations requires further investigation. TRPV4, a calcium influx and stress sensor channel, was also found to be overexpressed in PTC and the G-protein related signaling pathways were enriched in PTC.

Stress-related signaling pathways were found to be highly enriched in PTC compared with that in adjacent normal tissue, including cell adhesion (GO: 0007155), ECM organization (GO: 0030198), anatomical structure development (GO: 0048856), single-organism cellular process (GO: 0044763), response to stress (GO: 0006950) and cell proliferation (GO: 0008283). Although these pathways play important roles in several other types of tumors $(23,25)$; however, reports on their functions in PTC are limited. The present study provided a novel method with which to identify the factors affecting PTC distribution and its association with invasiveness. 

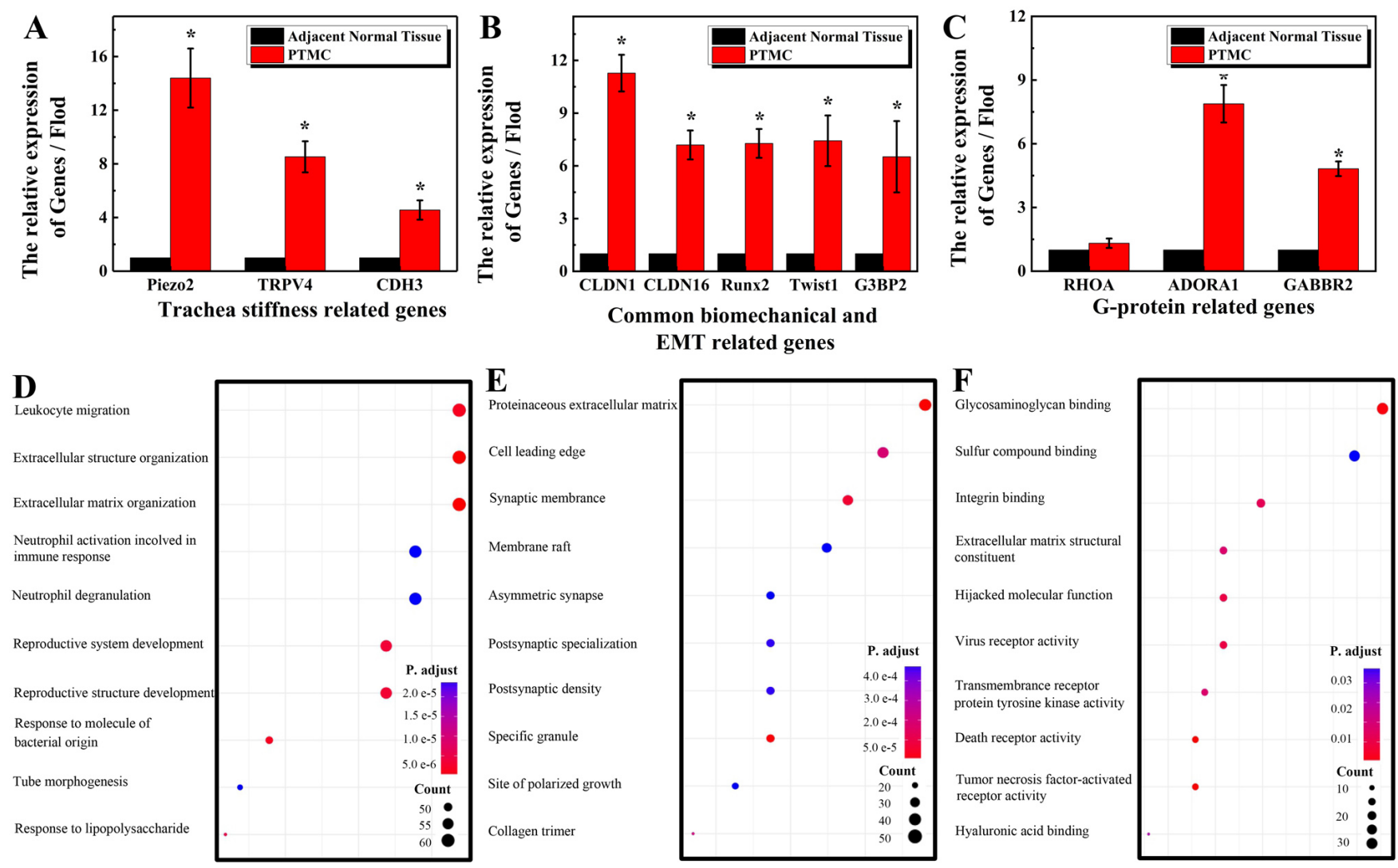

Figure 4. Differential gene expression and GO enrichment for PTC and paired normal tissue. mRNA expression levels of (A) trachea stiffness related genes (B) ECM stiffness and EMT related genes (C) G-protein-related genes. Top $10 \mathrm{GO}$ enrichment terms for (D) biological process, (E) cellular component and (F) molecular function. " $\mathrm{P}<0.05$, comparing to adjacent normal tissue. ECM, extracellular matrix.
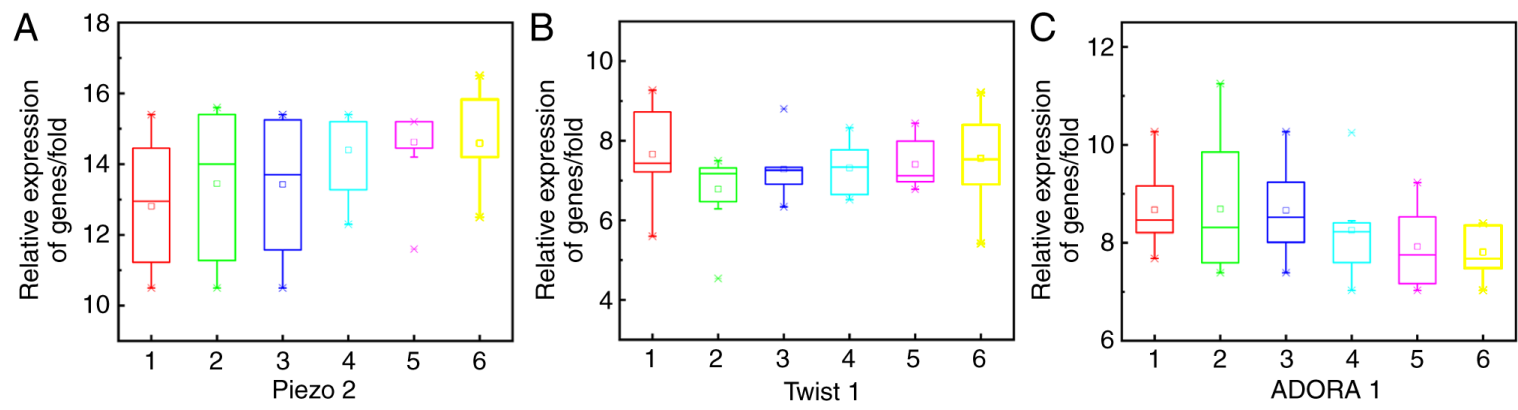

Figure 5. mRNA expression levels of Pizeo2 (A), Twistl (B) and ADORA1 (C). The 1-6 values on the x-axis represents 'Male', 'Female', 'Age, $\geq 50$ ', 'Age, <50', 'Thyroiditis' and 'No-thyroiditis', respectively.

In conclusion, the findings of the present study indicated that tracheal stiffness may exert a biomechanical effect on the thyroid; thus, may effect PTC distribution, providing a novel molecular mechanism and fundamental basis for the prediction and the development of possible novel treatment strategies for PTC.

\section{Acknowledgements}

Not applicable.

\section{Funding}

This study was support by Excellent Young Talents Fund Program of TJMUCH (grant no. 2019-1-11) and National
Natural Science Foundation of China (grant nos. 31400801 and 11572043).

\section{Availability of data and materials}

The datasets generated and/or analyzed during the current study are not publicly available, due to local legislation prohibiting data sharing in public databases, but are available from the corresponding author on reasonable request.

\section{Authors' contributions}

PL, RJ and $\mathrm{BH}$ designed the experiments, proposed the study, conducted the experiments, analyzed the data, wrote 
and edited the manuscript. HZ collected the clinical data from patients and the laboratory. TL and QL conducted the finite element analysis. XD conducted the RNA-sequencing and reverse transcription-quantitative PCR experiments and analyzed the data. All authors read and approved the final manuscript and agree to be accountable for all aspects of the research in ensuring that the accuracy or integrity of any part of the work are appropriately investigated and resolved. PL and RJ confirm the authenticity of all the raw data.

\section{Ethics approval and consent to participate}

The present study was approved by The Research Ethics Committee at Tianjin Medical University Cancer Hospital (Tianjin, China; approval no. 2018090). Written informed consent was provided by all the patients.

\section{Patient consent for publication}

Not applicable.

\section{Competing interests}

The authors declare that they have no competing interests.

\section{References}

1. Brooks SA, Lomax-Browne HJ, Carter TM, Kinch CE and Hall DM: Molecular interactions in cancer cell metastasis. Acta Histochem 112: 3-25, 2010.

2. Kausch I and Böhle A: Molecular aspects of bladder cancer III. Prognostic markers of bladder cancer. Eur Urol 41: 15-29, 2002.

3. Zhang R, Ma M, Dong G, Yao RR, Li JH, Zheng QD, Dong YY, $\mathrm{Ma} \mathrm{H}, \mathrm{Gao} \mathrm{DM}$, Cui JF, et al: Increased matrix stiffness promotes tumor progression of residual hepatocellular carcinoma after insufficient heat treatment. Cancer Sci 108: 1778-1786, 2017.

4. Levental KR, Yu H, Kass L, Lakins JN, Egeblad M, Erler JT, Fong SF, Csiszar K, Giaccia A, Weninger W, et al: Matrix crosslinking forces tumor progression by enhancing integrin signaling. Cell 139: 891-906, 2009.

5. Sung H, Ferlay J, Siegel RL, Laversanne M, Soerjomataram I, Jemal A and Bray F: Global cancer statistics 2020: GLOBOCAN estimates of incidence and mortality worldwide for 36 cancers in 185 countries. CA Cancer J Clin: Feb 4, 2021 (Epub ahead of print).

6. La Vecchia C, Malvezzi M, Bosetti C, Garavello W, Bertuccio P, Levi $\mathrm{F}$ and Negri E: Thyroid cancer mortality and incidence: A global overview. Int J Cancer 136: 2187-2195, 2015.

7. Liang W and Sun F: Identification of key genes of papillary thyroid cancer using integrated bioinformatics analysis. J Endocrinol Invest 41: 1237-1245, 2018.

8. Cui D, Zhao Y and Xu J: Activated CXCL5-CXCR2 axis promotes the migration, invasion and EMT of papillary thyroid carcinoma cells via modulation of $\beta$-catenin pathway. Biochimie 148: 1-11,2018

9. Jasim S, Baranski TJ, Teefey SA and Middleton WD: Investigating the effect of thyroid nodule location on the risk of thyroid cancer. Thyroid 30: 401-407, 2020.

10. Brauckhoff $M$ and Dralle H: Cervicovisceral resection in invasive thyroid tumors. Chirurg 80: 88-98, 2009 (In German).

11. Machens A, Hinze R and Dralle H: Surgery on the cervicovisceral axis for invasive thyroid cancer. Langenbecks Arch Surg 386: 318-323, 2001.

12. Kim JW, Roh JL, Gong G, Cho KJ, Choi SH, Nam SY and Kim SY: Treatment outcomes and risk factors for recurrence after definitive surgery of locally invasive well-differentiated papillary thyroid carcinoma. Thyroid 26: 262-270, 2016.

13. He S, Liu C, Li X, Ma S, Huo B and Ji B: Dissecting collective cell behavior in polarization and alignment on micropatterned substrates. Biophys J 109: 489-500, 2015.

14. Yao P and Xin WH:: Experimental study on the goiter's effect on the stress relaxation of thyroid. Chin J Control Endemic Dis 19: 79-80, 2004.
15. Trabelsi O, del Palomar AP, López-Villalobos JL, Ginel A and Doblaré M: Experimental characterization and constitutive modeling of the mechanical behavior of the human trachea. Med Eng Phys 32: 76-82, 2010.

16. Haddad RI, Nasr C, Bischoff L, Busaidy NL, Byrd D, Callender G, Dickson P, Duh QY, Ehya H, Goldner W, et al: NCCN guidelines insights: Thyroid carcinoma, version 2.2018. J Natl Compr Canc Netw 16: 1429-1440, 2018.

17. Cabanillas ME, McFadden DG and Durante C: Thyroid cancer. Lancet 388: 2783-2795, 2016.

18. Robinson MD, Mccarthy DJ and Smyth GK: edgeR: A bioconductor package for differential expression analysis of digital gene expression data. Bioinformatics 26: 139-140, 2010.

19. Benjamini Y and Hochberg Y: Controlling the false discovery rate: A practical and powerful approach to multiple testing. J R Statist Soc B 57: 289-300, 1995.

20. Szklarczyk D, Franceschini A, Kuhn M, Simonovic M, Roth A, Minguez P, Doerks T, Stark M, Muller J, Bork P, et al: The STRING database in 2011: Functional interaction networks of proteins, globally integrated and scored. Nucleic Acids Res 39 (Database Issue): D561-D568, 2011.

21. Adamcsek B, Palla G, Farkas IJ, Derényi I and Vicsek T: CFinder: Locating cliques and overlapping modules in biological networks. Bioinformatics 22: 1021-1023, 2006.

22. Livak KJ and Schmittgen TD: Analysis of relative gene expression data using real-time quantitative PCR and the 2(-Delta Delta C(T)) method. Methods 25: 402-408, 2001.

23. Peng Y, Chen Y, Qin X, Li S and Liu Y: Unveiling the mechanotransduction mechanism of substrate stiffness-modulated cancer cell motility via ROCK1 and ROCK2 differentially regulated manner. FASEB J 33: 644.4, 2019.

24. Liang J, Zhang XL, Yuan JW, Zhang HR, Liu D, Hao J, Ji W, Wu XZ and Chen D: Cucurbitacin B inhibits the migration and invasion of breast cancer cells by altering the biomechanical properties of cells. Phytother Res 33: 618-630, 2019.

25. Pardo-Pastor C, Rubio-Moscardo F, Vogel-González M, Serra SA, Afthinos A, Mrkonjic S, Destaing O, Abenza JF, Fernández-Fernández JM, Trepat X, et al: Piezo2 channel regulates RhoA and actin cytoskeleton to promote cell mechanobiological responses. Proc Natl Acad Sci USA 115: 1925-1930, 2018.

26. McKenzie AJ, Hicks SR, Svec KV, Naughton H, Edmunds ZL and Howe AK: The mechanical microenvironment regulates ovarian cancer cell morphology, migration, and spheroid disaggregation. Sci Rep 8: 7228, 2018

27. Woo SH, Lukacs V, de Nooij JC, Zaytseva D, Criddle CR, Francisco A, Jessell TM, Wilkinson KA and Patapoutian A: Piezo2 is the principal mechanotransduction channel for proprioception. Nat Neurosci 18: 1756-1762, 2015.

28. Coste B, Mathur J, Schmidt M, Earley TJ, Ranade S, Petrus MJ, Dubin AE and Patapoutian A: Piezo1 and Piezo2 are essential components of distinct mechanically activated cation channels. Science 330: 55-60, 2010.

29. Ferrari LF, Bogen O, Green P and Levine JD: Contribution of Piezo2 to endothelium-dependent pain. Mol Pain 11: 65, 2015.

30. Nonomura K, Woo SH, Chang RB, Gillich A, Qiu Z, Francisco AG, Ranade SS, Liberles SD and Patapoutian A: Piezo2 senses airway stretch and mediates lung inflation-induced apnoea. Nature 541: 176-181, 2017.

31. Yang H, Liu C, Zhou RM, Yao J, Li XM, Shen Y, Cheng H, Yuan J, Yan B and Jiang Q: Piezo2 protein: A novel regulator of tumor angiogenesis and hyperpermeability. Oncotarget 7: 44630-44643, 2016.

32. Kameda Y, Nishimaki T, Chisaka O, Iseki S and Sucov HM: Expression of the epithelial marker E-cadherin by thyroid $\mathrm{C}$ cells and their precursors during murine development. J Histochem Cytochem 55: 1075-1088, 2007.

33. Taniuchi K, Nakagawa H, Hosokawa M, Nakamura T, Eguchi H, Ohigashi $\mathrm{H}$, Ishikawa $\mathrm{O}$, Katagiri $\mathrm{T}$ and Nakamura $\mathrm{Y}$ : Overexpressed P-cadherin/CDH3 promotes motility of pancreatic cancer cells by interacting with p120ctn and activating rho-family GTPases. Cancer Res 65: 3092-3099, 2005.

34. Albergaria A, Resende C, Nobre AR, Ribeiro AS, Sousa B, Machado JC, Seruca R, Paredes J and SchmittF: CCAAT/enhancer binding protein $\beta(\mathrm{C} / \mathrm{EBP} \beta)$ isoforms as transcriptional regulators of the pro-invasive $\mathrm{CDH} / \mathrm{P}$-cadherin gene in human breast cancer cells. PLoS One 8: e55749, 2013.

This work is licensed under a Creative Commons Attribution-NonCommercial-NoDerivatives 4.0 International (CC BY-NC-ND 4.0) License. 